\title{
JOURNAL OF RHFOLOGY
}

Rheology of non-Brownian particles suspended in concentrated colloidal dispersions at low particle Reynolds number

Colin D. Cwalina and Norman J. Wagner

Citation: Journal of Rheology 60, 47 (2016); doi: 10.1122/1.4935445

View online: http://dx.doi.org/10.1122/1.4935445

View Table of Contents: http://scitation.aip.org/content/sor/journal/jor2/60/1?ver=pdfcov

Published by the The Society of Rheology

\section{Articles you may be interested in}

Flows of suspensions of particles in yield stress fluids

J. Rheol. 59, 1449 (2015); 10.1122/1.4934363

An effective medium approach for the elongational viscosity of non-colloidal and non-Brownian hard-sphere suspensions

Phys. Fluids 27, 083304 (2015); 10.1063/1.4928974

Dynamics of the orientation behavior and its connection with rheology in sheared non-Brownian suspensions of anisotropic dicolloidal particles

J. Rheol. 55, 581 (2011); 10.1122/1.3569585

Transient response of magnetorheological fluids: Shear flow between concentric cylinders

J. Rheol. 49, 87 (2005); 10.1122/1.1803576

Orientational order in concentrated dispersions of plate-like kaolinite particles under shear

J. Rheol. 44, 221 (2000); 10.1122/1.551093

\section{圆 Re-register for Table of Content Alerts}




\title{
Rheology of non-Brownian particles suspended in concentrated colloidal dispersions at low particle Reynolds number
}

\author{
Colin D. Cwalina and Norman J. Wagner ${ }^{\text {a) }}$ \\ Center for Molecular Engineering and Thermodynamics, Department of Chemical and Biomolecular Engineering, \\ University of Delaware, Newark, Delaware 19716
}

(Received 3 August 2015; final revision received 15 October 2015; published 8 December 2015)

\begin{abstract}
The shear flow of non-Brownian glass spheres suspended in a concentrated colloidal dispersion that exhibits non-Newtonian rheology is investigated. At low volume fractions, the addition of non-Brownian spherical particles to the colloidal dispersion leads to an increase in the steady shear viscosity as well as the dynamic moduli. The flow curves of these suspensions are qualitatively similar to the suspending colloidal dispersion medium, and as such, in this semidilute regime, the suspension data can be shifted on to that of the colloidal dispersion medium at constant shear stress with shift factors comparable to those predicted for spherical particles in a Newtonian fluid. At higher volume fractions of non-Brownian spheres, the shear thickening power law exponent increases with the addition of non-Brownian particles. This increase in the shear thickening power law exponent is shown to be consistent with the effects of confinement on the shear thickening colloidal dispersion by the larger non-Brownian particles. @ 2016 The Society of Rheology. [http://dx.doi.org/10.1122/1.4935445]
\end{abstract}

\section{INTRODUCTION}

Rigid spherical particles immersed in a fluid acquire translational and rotational motion as a result of an imposed bulk laminar shear flow. The fluid flow between particles in a flowing suspension is complex in comparison to laminar flow. However, for creeping shear flow with a Newtonian suspending fluid, a universality in the flow behavior exists such that the suspension viscosity is independent of the particle size and size distribution, and only a function of the volume fraction relative to maximum packing [1]. Einstein [2] calculated the particle contribution to the viscosity in the dilute limit as the linear term in an expansion in particle volume fraction

$$
\eta_{\mathrm{r}}=1+2.5 \phi+\mathrm{k}_{2} \phi^{2}+\cdots,
$$

where $\eta_{\mathrm{r}}$ is the relative viscosity of the suspension and $\phi$ is the volume fraction of spherical particles. Additional terms are needed to account for the contribution of particle interactions to the viscosity. For the case of a randomized microstructure, Batchelor and Green [3] calculated the value of $k_{2}$ to be 5.2 for monodisperse hard spheres, which was later refined to a value of 5.0 by Wagner and Woutersen [4]. A plethora of models have been proposed for more concentrated suspensions (see, for example, a recent comparison by Faroughi and Huber [5]). In particular, the semiempirical model of Morris and Boulay [6] can correlate the measured and simulated viscosity and normal stress differences, with coefficients recently reported by Cwalina and Wagner [7]. Including Brownian motion introduces a time scale and the

\footnotetext{
a) Author to whom correspondence should be addressed; electronic mail: wagnernj@udel.edu
}

viscosity becomes dependent on the Péclet number, such that the low and high shear viscosities, as the well as the viscosity in the shear-thickened state can be defined [8] and are successfully described by semiempirical models [7,9]. This very brief introduction demonstrates that extensive research has mapped out the rheological properties of suspensions of hard spheres in Newtonian fluids with and without Brownian motion, although much remains to be determined concerning the effects of size polydispersity [10,11], particle shape, the presence of any additional interparticle interactions such as friction [12], particle inertia [13], as well as the effects of shear-induced particle migration and shear banding [14].

Many real-world industrial processes involve the flow of suspensions of non-Brownian particles suspended in colloidal dispersions. Concrete-formed by mixing cement with water, sand, and gravel-is the most widely consumed construction material in the world [15]. Cement itself is comprised of colloidal particles that exhibit shear thinning and shear thickening rheology [16]. Highways in the United States are made almost exclusively out of Portland cement concrete along with asphalt and bitumen wearing surfaces [17]. In the energy sector, concentrated coal-water slurries are being investigated as replacements for petroleum-derived fuels [18]. Transporting these slurries through long pipelines has motivated a recent surge in the study of their nonNewtonian flow properties [19]. Additionally, large quantities of mineral tailings from mining operations must be pumped to disposal facilities where knowledge of nonNewtonian flow behavior is essential for transport optimization [20]. On a more tasteful level, ice cream is formed from a liquid suspension containing tiny ice crystals and fat globules in a syrup of sugars and polysaccharides [21]. These applications are representative of the broad use of suspensions comprised of both Brownian and non-Brownian particles, which will benefit from a basic, quantitative understanding of 
how the flow properties of a non-Newtonian suspending medium comprised of a colloidal dispersion will be affected by the addition of non-Brownian particles.

Despite comprising some of the most significant materials used by mankind in terms of mass and volume, there is a notable absence in the literature of systematic studies of model suspensions of non-Brownian particles in shear thickening colloidal dispersions. Liard et al. [22] studied suspensions of $>50 \mu \mathrm{m}$ glass beads in a shear thickening suspension of cornstarch and water, but the cornstarch particles were outside of the colloidal size range. Recent measurements were also made in pressure-driven rheometry on suspensions of non-Brownian spheres in yield stress emulsions [23]. In contrast, there exists significant research into the rheology of suspensions of non-Brownian particles in viscoelastic fluids, such as polymer and surfactant solutions and polymer melts (for a review, see [1]). The nonNewtonian nature of the suspending fluid has been shown to directly affect particle motion [24-29]. The fore-aft symmetry between two approaching particles is lost [30], which can lead to a number of surprising microstructural changes for dilute suspensions under shear flow, including particle chaining [31-33]. With regards to shear thickening colloidal dispersions, a recent report documents the rheological and morphological properties of "suspoemulsions," where at high colloidal dispersion concentrations, the multiphase fluid can exist as an emulsion suspended in a shear thickening fluid [34]. Here, the presence of a low viscosity emulsion within the colloidal dispersion systematically shifts the non-Newtonian shear rheology, and an important observation is that the shear thickening transition for the suspoemulsions scales more closely with the applied shear stress than the shear rate.

Direct numerical simulation results for spherical particles suspended in a viscoelastic medium are available for a number of constitutive models for the suspending fluid, but such simulations are generally limited to investigating the detailed motion of a small number of particles. The large disparity in time and length scales required for a direct particle simulation of a suspension of non-Brownian spheres in a dispersion of Brownian spheres that properly includes hydrodynamic interactions is beyond current computational capabilities [35]. Note that such systems cannot be merely viewed in terms of particle size polydispersity. The treatment of a suspension of non-Brownian particles mixed with Brownian particles requires simulating particles separated by an order of magnitude or more difference in length scales, which translates into over three orders of magnitude in mass for equivalent densities. We also note that the effect of mixing binary size mixtures of stable colloidal particles is understood in terms of hydrodynamic effects [36], whereas binary mixtures of non-Brownian particles are also well understood in terms of the dependence on the maximum packing fraction $[37,38]$.

Remarkably, despite the complex local flows between particles in suspensions, a plethora of experimental evidence shows the bulk viscosity of suspensions of non-Brownian spherical particles in viscoelastic fluids largely reflects the non-Newtonian viscosity of the suspending medium, and as a result, scaling laws have been proposed. Early work on polymer solutions by Highgate and Whorlow [39] first suggested that relative fluidities of suspensions could be collapsed on to that of the suspending fluid with simple shift factors over a wide range of particle fractions. Additional authors [40-47] investigated other types of viscoelastic suspending fluids and observed similar behavior. Using an effective shear rate concept, Ohl and Gleissle [48] formalized a general shifting procedure to collapse suspension data on to that of the suspending medium at a constant shear stress

$$
\text { Shift Factor }=\left.\frac{\dot{\gamma}(\phi=0)}{\dot{\gamma}(\phi)}\right|_{\sigma}=\left.\frac{\eta(\phi)}{\eta(\phi=0)}\right|_{\sigma} .
$$

This shifting procedure works remarkably well to collapse suspension data across a broad range of volume fractions and for various types of viscoelastic media. It is important to note that the premise for this shifting procedure is the treatment of the viscoelastic medium as a continuous fluid phase. Although not formulated specifically for analyzing suspensions of non-Brownian particles in colloidal dispersions, this body of literature provides a possible framework in the absence of other theories.

In the present work, we explore and report on the shear flow behavior of non-Brownian spherical particles suspended in model, non-Newtonian colloidal dispersions. This particular choice of suspending medium is of particular interest in that colloidal dispersions exhibit a rich shear-rate dependent rheology that includes a zero-shear viscosity [9], viscoelasticity [49], shear thinning, a high shear viscosity plateau, and shear thickening at high shear rates $[7,50]$. In this study, the non-Brownian and colloidal particles are separated by more than an order of magnitude in size and more than three orders of magnitude in mass. As such, we explore treating the colloidal dispersion as a continuous fluid and test the applicability of the viscosity shifting hypothesis of Ohl and Gleissle [48] to this particular class of suspensions in nonNewtonian fluids. The dynamic moduli and normal stress differences are also investigated and their behavior compared to that observed for suspensions of non-Brownian particles in various other viscoelastic media.

\section{EXPERIMENTAL SECTION}

\section{A. Rheological characterization}

Rheological measurements were performed on a torquecontrolled Discovery Hybrid Rheometer from TA Instruments (New Castle, DE). A $20 \mathrm{~mm}$ parallel plate tool was used for steady shear and dynamic oscillatory measurements. A cone and plate tooling was also studied, but difficulties were encountered due to the large particle size relative to the truncation gap. In steady shear measurements, the shear stress, $\sigma$, was extracted from Eq. (3) as

$$
\sigma=\frac{M}{2 \pi R^{3}}\left[3+\frac{d \ln M}{d \ln \dot{\gamma}_{R}}\right]
$$


where $M$ is the applied torque, $R$ is the plate radius, and $\dot{\gamma}_{R}$ is the shear rate at the rim of the plate. The difference between the first and second normal stress differences, $\mathrm{N}_{1}$ and $\mathrm{N}_{2}$, respectively, was obtained as

$$
\mathrm{N}_{1}-\mathrm{N}_{2}=\frac{F_{Z}}{\pi R^{2}}\left[2+\frac{d \ln F_{z}}{d \ln \dot{\gamma}_{R}}\right]
$$

where $F_{Z}$ is the axial thrust on the tool, and $\dot{\gamma}_{R}$ is the shear rate at the rim. In oscillatory measurements, the dynamic moduli were computed as

$$
\begin{aligned}
\mathrm{G}^{\prime}(\omega) & =\frac{2 H M \cos \delta}{\pi R^{4} \theta}, \\
\mathrm{G}^{\prime \prime}(\omega) & =\frac{2 H M \sin \delta}{\pi R^{4} \theta},
\end{aligned}
$$

where $H$ is the gap height, $\delta$ is the phase angle, and $\theta$ is the angular displacement [51].

Samples were loaded to a gap height of $300 \mu \mathrm{m}$ using a force-gap control with a constant rate of $5 \mu \mathrm{m} / \mathrm{s}$ and a maximum allowable axial force of $0.5 \mathrm{~N}$. Frequency sweeps were performed at $1 \%$ strain and data acquired over 10 cycles. In steady shear experiments, at a given applied torque, $10 \mathrm{~s}$ were allowed for equilibration, and the viscosity and normal force were recorded over the following $60 \mathrm{~s}$. Particle migration can be a significant complication for the measurement of non-Brownian suspensions at high shear rates. However, in our work, the frequency sweeps and steady flow curves were found to be reversible, indicating that particle migration and sedimentation were negligible on the timescale of our measurements. Slip measurements were made previously [7] on one of the colloidal dispersions considered in this work, and wall slip was shown to be insignificant with regard to the measured flow curves.

\section{B. Materials}

Two different colloidal dispersions consisting of similar particles varying in size were formulated as suspending fluids. The first consisted of silica nanoparticles (Seahostar KE-P10, Nippon Shokubai Co., Tokyo, Japan) dispersed in a polyethylene glycol (PEG) $\mathrm{MW}=200$ (Aldrich Chemical Company, Allentown, PA; $\eta_{f}=0.05 \mathrm{~Pa}^{*} \mathrm{~s}, \quad \rho=1.12 \mathrm{~g} / \mathrm{cm}^{3}$ ) solvent. Small angle neutron scattering found the particle radius of these KE-P10 particles to be $a=60 \mathrm{~nm}$ and the particle density determined from densitometry was $\rho_{\mathrm{p}}=1.89 \mathrm{~g} / \mathrm{cm}^{3}$ [52]. The second suspending fluid consisted of silica nanoparticles (Seahostar KE-P50, Nippon Shokubai Co., Tokyo, Japan) of radius $a=260 \mathrm{~nm}$ and particle density, $\rho_{\mathrm{p}}=1.96 \mathrm{~g} / \mathrm{cm}^{3}$ [52] in a PEG-200 solvent. The colloidal dispersions were prepared by roll mixing for one week, and the suspending fluid compositions are reported in Table I.

The non-Newtonian behavior of hard-sphere colloidal dispersions is the result of a competition between thermodynamic Brownian forces and hydrodynamic forces resulting from the imposed shear flow that leads to shear-induced changes in the microstructure. This has been extensively characterized for these colloidal dispersions by rheology and
TABLE I. Composition of suspending fluids.

\begin{tabular}{lccc}
\hline \hline Dispersion & Wt. \% Solids & $\rho\left(\mathrm{g} / \mathrm{cm}^{3}\right)$ & $\phi$ \\
\hline KE-P10 in PEG-200 & 50 & 1.41 & 0.37 \\
KE-P50 in PEG-200 & 54 & 1.46 & 0.40 \\
\hline \hline
\end{tabular}

small angle neutron scattering under flow [53]. The appropriate dimensionless group to gauge this competition is the Péclet number $(\mathrm{Pe})$ given by

$$
P e=\frac{6 \pi \eta_{f} \dot{\gamma} a^{3}}{k T},
$$

where $\eta_{f}$ is the viscosity of the suspending fluid, $\dot{\gamma}$ is the shear rate, $a$ is the particle radius, $k$ is the Boltzmann constant, and $T$ is the absolute temperature. For oscillatory measurements, the shear rate in Eq. (7) can be replaced by the product of the strain amplitude and the frequency $\left(\dot{\gamma} \rightarrow \gamma_{o} \omega\right)$. Measurement across a broader range of $P e$ number was achieved by recognizing that $P e$ scales as $a^{3}$ and that the Brownian stress, and hence elasticity, scales inversely with $a^{3}$ [54]. Thus, the use of two colloidal dispersions with widely varying particle sizes as suspending fluids enables exploring a broad range of non-Newtonian phenomena associated with near hard-sphere dispersions, namely, viscoelasticity at low $P e$ and shear thickening at large $P e$. This also enables probing the effects of size ratio upon the addition of non-Brownian particles. The flow curves of both colloidal dispersions are shown together in Fig. 1 as a function of the $\mathrm{Pe}$ number. The use of the two different dispersions enables exploring the non-Newtonian behavior of near hard-spheres dispersions over 8 orders of magnitude in $P e$ number within the measurement range of our rheometer and tooling. The KE-

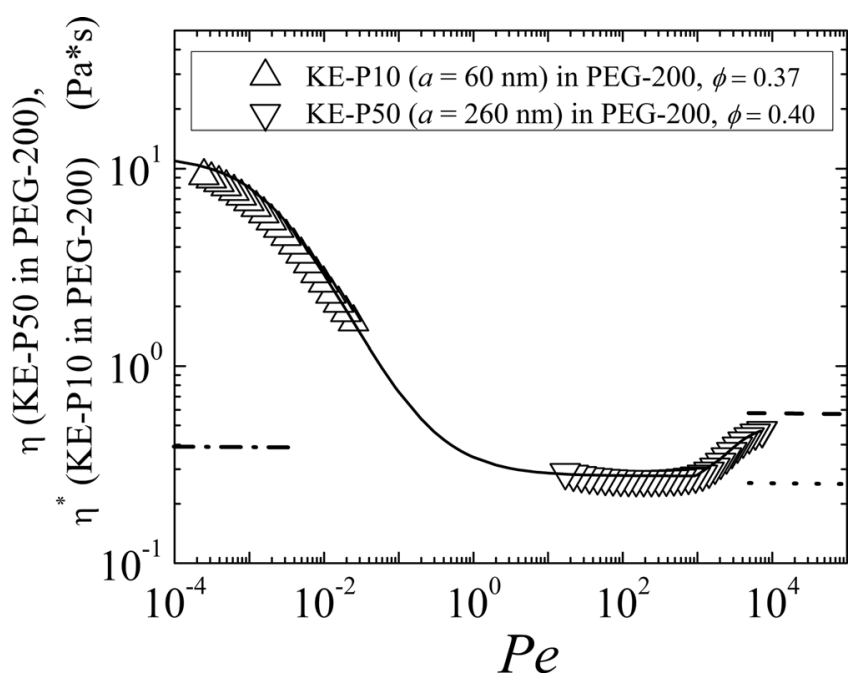

FIG. 1. Steady and complex viscosity as a function of the $P e$ number for the colloidal dispersions used as suspending fluids in this work. The dashed line is the predicted value of the shear-thickened state viscosity for a $\phi=0.40$ near hard-sphere colloidal dispersion [7], the dotted line is the limiting value of the high shear plateau viscosity for a $\phi=0.40$ hard-sphere dispersion [57], and the dashed-dotted line is the limiting value of the zero-shear viscosity for a $\phi=0.37$ dispersion calculated using the analysis of [56]. 
P10 in PEG-200 dispersion has measureable viscoelastic moduli while the KE-P50 in PEG-200 dispersion allows exploring the high shear viscosity plateau and shear thickening behavior at higher $P e$ number. At the largest $P e$ numbers explored in this study, the viscosity tends toward a plateau value. This plateau in the viscosity is the emergence of the colloidal shearthickened state, predicted from theory $[8,55]$, and recently confirmed to exist by the experiments in [7]. The suspending colloidal fluids are compared in Fig. 1 to expected hard-sphere behavior through the values of the zero-shear viscosity [56], the high shear plateau viscosity [57], and the viscosity in the shear-thickened state [7]. The values of the high shear plateau and shear-thickened state viscosities for the $\phi=0.40$ $(a=260 \mathrm{~nm})$ dispersion are very close to the expected limiting hard-sphere behavior. The viscosity curve of the $\phi=0.37$ $(a=60 \mathrm{~nm})$ dispersion clearly tends toward a zero-shear viscosity above the limiting hard-sphere value. Nearly identical viscosity measurements were obtained on this exact dispersion and volume fraction by [52], and attractive interactions between these particles are likely the source of this deviation from ideal hard-sphere behavior.

The non-Brownian spherical particles used in this study were polydisperse $\left(\mathrm{d}_{10}=5 \mu \mathrm{m}, \mathrm{d}_{50}=10 \mu \mathrm{m}\right.$, and $\mathrm{d}_{90}=21 \mu \mathrm{m}$ ) hollow fused borosilicate glass (Sphericel $^{\circledR}$ 110P8, Potters Industries LLC, Valley Forge, PA) with a manufacturer reported particle density, $\rho_{\mathrm{p}}=1.10 \mathrm{~g} / \mathrm{cm}^{3}$. The non-Brownian particles (abbreviated "NBP" in subscripts to follow) are nearly density matched to the PEG-200 such that gravitational settling is not a factor on the timescale over which rheological characterization takes place. The nonBrownian particles have an average diameter 20 times that of the KE-P50 particles and are 4000 times more massive. Additionally, they are approximately 80 times larger and 300,000 times more massive than the KE-P10 particles. Suspensions were prepared by the addition of the nonBrownian particles to the colloidal dispersions and roll mixing for one week. The suspensions were formulated by weight and the volume fractions calculated using the measured densities. Throughout the remainder of this paper, the volume fraction of non-Brownian particles, $\phi_{\mathrm{NBP}}$, is calculated as the volume of non-Brownian particles divided by the total volume of the suspension, noting that the volume fraction of the colloidal particles in the suspending liquid medium remains constant. The suspension compositions studied are reported in Table II.

Although the particles were nearly neutrally buoyant, particle inertia can create shear thickening for a particle Reynolds number $\left(R e_{p}\right)$ on the order of $10^{-1}$ or larger [59]. The particle Reynolds number is defined using the particle radius as the relevant length scale

$$
R e_{p}=\frac{\dot{\gamma} a^{2} \rho}{\eta_{f}} .
$$

During rheological measurement, the largest particle Reynolds number encountered in any of the suspensions was on the order of $10^{-3}$ so particle inertia was not a contributing factor to the measured suspension viscosity.
TABLE II. Suspension compositions used in this study.

\begin{tabular}{|c|c|c|c|c|c|}
\hline \multicolumn{6}{|c|}{ Suspending Fluid: KE-P10 $(a=60 \mathrm{~nm})$ in PEG-200 $(\phi=0.37)$} \\
\hline Suspension & Wt. \% NBP & $\rho\left(\mathrm{g} / \mathrm{cm}^{3}\right)$ & $\phi_{\mathrm{NBP}}$ & $\phi_{\mathrm{KE}-\mathrm{P} 10}$ & $\phi_{\text {Total Solids }}$ \\
\hline 1 & 4 & 1.41 & 0.05 & 0.35 & 0.40 \\
\hline 2 & 8 & 1.40 & 0.10 & 0.33 & 0.43 \\
\hline 3 & 12 & 1.38 & 0.15 & 0.31 & 0.46 \\
\hline \multicolumn{6}{|c|}{ Suspending Fluid: KE-P50 $(a=260 \mathrm{~nm})$ in PEG-200 $(\phi=0.40)$} \\
\hline Suspension & Wt. \% NBP & $\rho\left(\mathrm{g} / \mathrm{cm}^{3}\right)$ & $\phi_{\mathrm{NBP}}$ & $\phi_{\mathrm{KE}-\mathrm{P} 50}$ & $\phi_{\text {Total Solids }}$ \\
\hline 1 & 3 & 1.44 & 0.04 & 0.39 & 0.43 \\
\hline 2 & 7 & 1.42 & 0.09 & 0.36 & 0.45 \\
\hline 3 & 12 & 1.40 & 0.15 & 0.34 & 0.49 \\
\hline 4 & 30 & 1.33 & 0.36 & 0.26 & 0.62 \\
\hline 5 & 38 & 1.30 & 0.45 & 0.22 & 0.67 \\
\hline 6 & 41 & 1.29 & 0.48 & 0.21 & 0.69 \\
\hline
\end{tabular}

\section{RESULTS AND DISCUSSION}

\section{A. Linear viscoelastic regime}

Brownian motion within a colloidal dispersion gives rise to viscoelasticity [49], which is evident in the dynamic moduli of the $\phi=0.37$ dispersion of KE-P10 in PEG-200 shown in Fig. 2. The effect of adding small amounts of nonBrownian spheres to the dispersion is also shown in this figure. At a given frequency, the addition of non-Brownian particles results in a vertical shift of both dynamic moduli, while the shape of the curve reflects that of the colloidal dispersion as the suspending medium. This result is consistent with measurements of the dynamic moduli in other viscoelastic suspending fluids [60-64]. As nondeformable spherical particles are added to the colloidal dispersion medium, the local strain amplitudes in the colloidal dispersion are, on average, greater than the applied strain amplitudes. Consequently, the dynamic stresses are higher, and, when divided by the applied deformation, yield higher moduli.

Visual inspection suggests that a single scalar shift should be sufficient to shift the dynamic moduli data onto that of the colloidal dispersion medium and form a master curve. Indeed, such a single vertical shift factor for a given volume

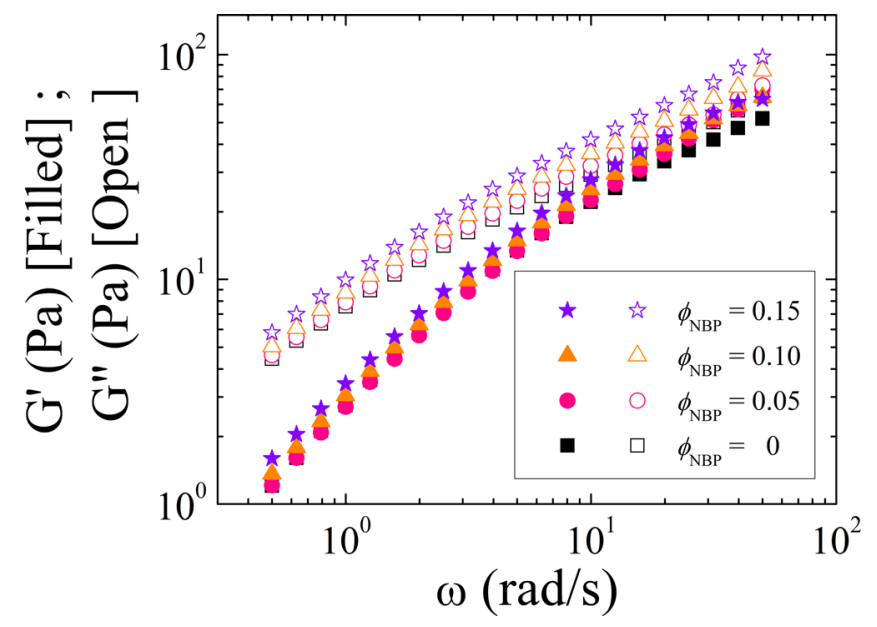

FIG. 2. Dynamic moduli of suspensions of non-Brownian glass spheres in a $\phi=0.37$ KE-P10:PEG-200 colloidal dispersion. 


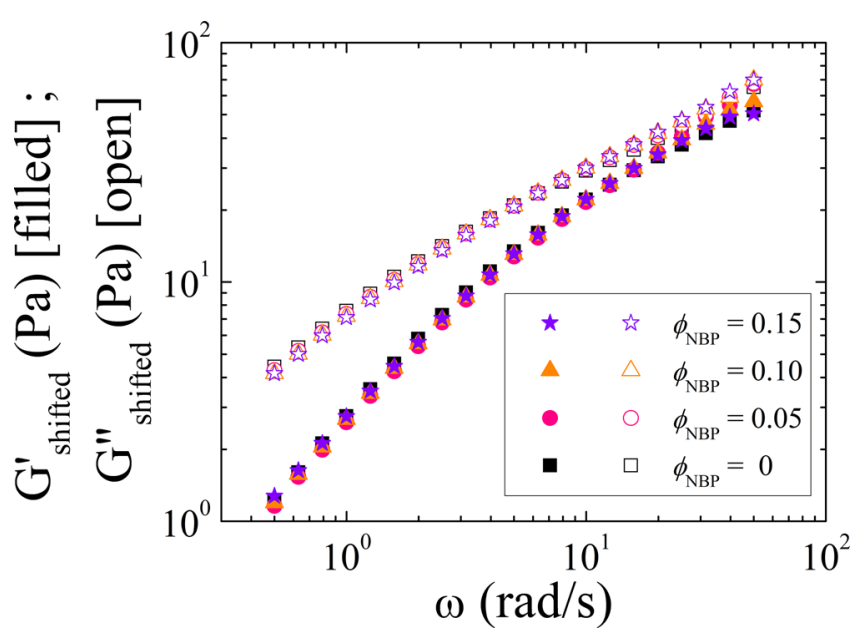

FIG. 3. Shifted dynamic moduli curves. Symbols are identical to those used in Fig. 2.

fraction of non-Brownian spheres successfully forms a master curve, as shown in Fig. 3. Furthermore, the dynamic moduli can be shifted onto the values for the colloidal dispersion medium with roughly the same shift factor, as reported in Fig. 4. This result has already been demonstrated for suspensions of non-Brownian spheres in Newtonian fluids where the suspension relative viscosity and elastic modulus have been shown to collapse onto a master curve when plotted against the proximity to maximum packing [1,37].

Figure 5 shows the moduli after the purely hydrodynamic component of the loss modulus $\left(\omega \eta_{\infty}^{\prime}\right)$ has been subtracted so as to show the contribution arising from Brownian and interparticle interactions. This enables identifying the characteristic relaxation time from the crossover frequency. The value of the crossover frequency is shown to be independent of the concentration of non-Brownian particles in this semidilute concentration regime. The high frequency viscosity relative to that of the underlying colloidal dispersion medium is plotted as a function of the volume fraction of nonBrownian particles in Fig. 6 along with the results expected

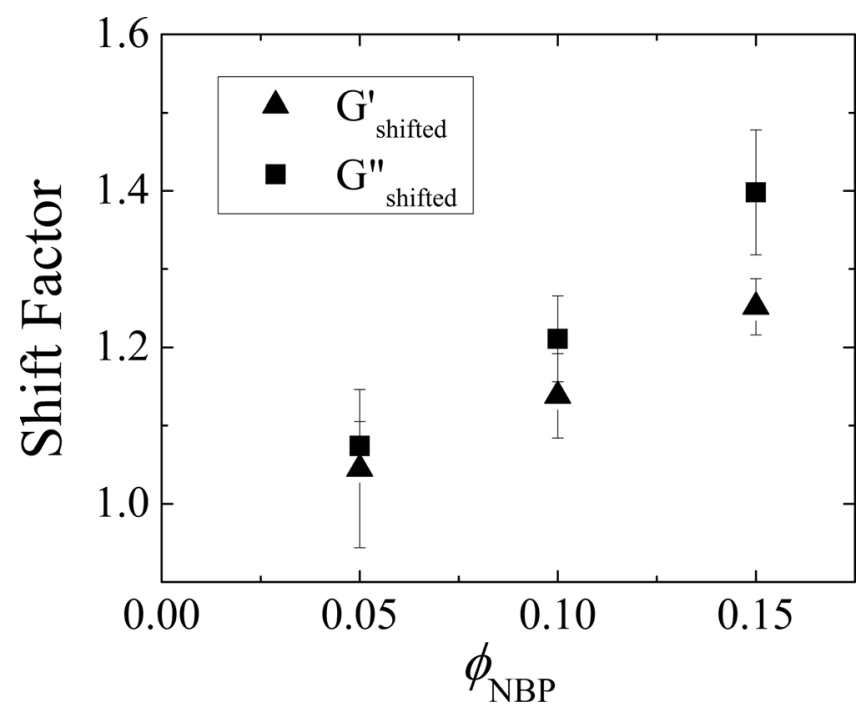

FIG. 4. Shift factors required to collapse dynamic moduli data onto that of the colloidal dispersion medium as a function of the volume fraction of nonBrownian spheres.

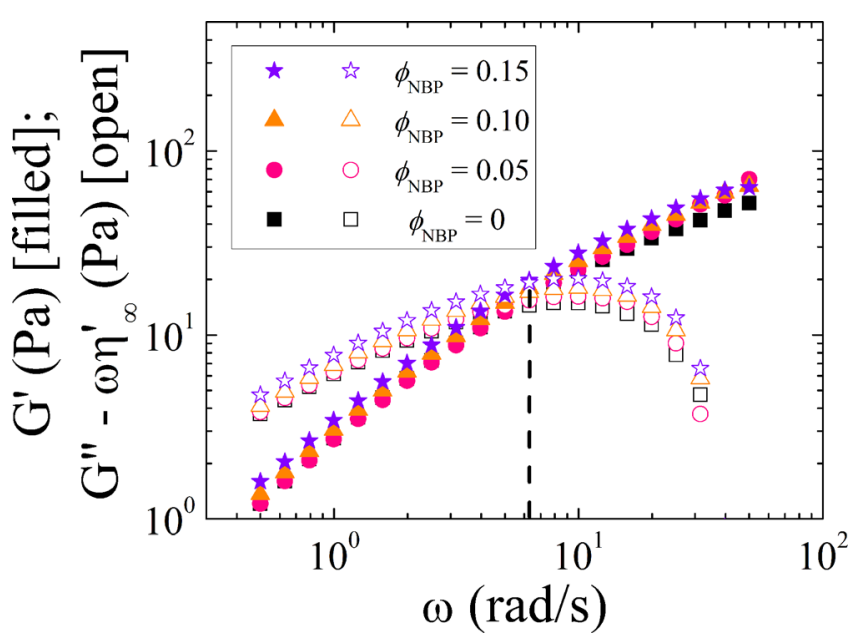

FIG. 5. Dynamic moduli of suspensions of non-Brownian glass spheres in a $\phi=0.37$ KE-P10 in PEG-200 colloidal dispersion with the hydrodynamic component of the loss modulus subtracted off. The dashed vertical line marks the crossover frequency, which is observed to be independent of the concentration of non-Brownian particles.

for non-Brownian particles in a Newtonian fluid [Eq. (1)]. As shown the high frequency viscosity for these suspensions in a non-Newtonian colloidal dispersion medium can be well approximated by Eq. (1) in the semidilute concentration regime.

\section{B. High shear plateau and shear thickening upturn: Semidilute regime}

To access the high shear plateau and a significant shear thickening regime within the measurement range of the rheometer, we studied the same non-Brownian spheres suspended in the colloidal dispersion comprised of the larger KE-P50 $(a=260 \mathrm{~nm})$ colloidal particles in PEG-200. The viscosity of suspensions over a range of concentrations of added non-Brownian particles is shown in Fig. 7.

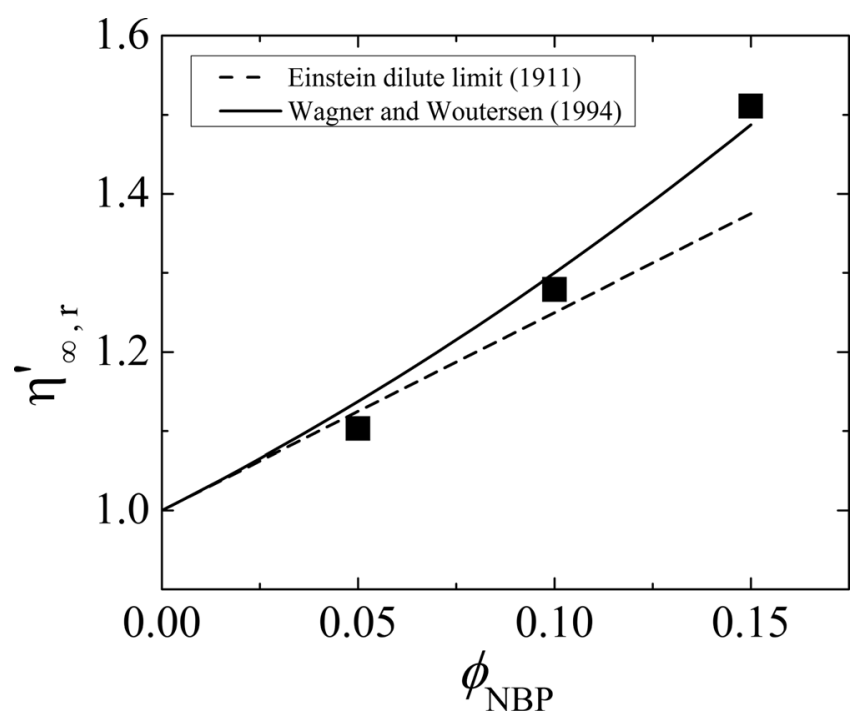

FIG. 6. Relative high frequency viscosity for non-Brownian glass spheres suspended in a $\phi=0.37$ KE-P10:PEG-200 colloidal dispersion. The dashed line is the Einstein viscosity equation [Eq. (1), $\mathrm{k}_{2}=0$ ] for dilute suspensions of non-Brownian spheres in a Newtonian fluid and the solid line contains the additional $\phi^{2}$ term of $\mathrm{k}_{2}=5.0$ [4]. 


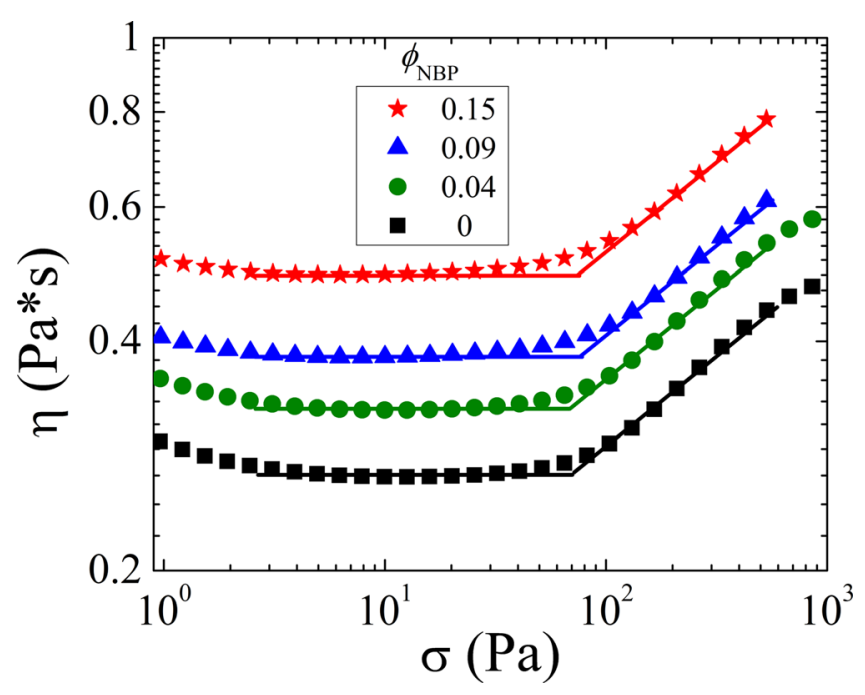

FIG. 7. Viscosity of non-Brownian glass spheres suspended in a shear thickening colloidal dispersion of KE-P50 $(a=260 \mathrm{~nm})$ particles in PEG-200 as a function of the shear stress. Solid lines are extensions to power law fits to the high shear plateau and shear thickening upturn.

By plotting the suspension viscosity as a function of shear stress, the addition of non-Brownian particles is observed to shift the viscosity curves vertically without a change in shape. Indeed, the exponent of the power law fits to the viscosity in the shear thickening regime remains constant. The critical stress for shear thickening is defined to be the intersection of the high shear plateau with this power law scaling. From Fig. 7, it is evident that the onset of shear thickening occurs at a nearly constant value of the shear stress independent of the volume fraction of non-Brownian spheres. The onset of shear thickening in dispersions of spherical colloids is well established to be stress-controlled $[36,65,66]$, and the data here suggest that the onset of shear thickening in suspensions of non-Brownian particles in these colloidal dispersions is also stress-controlled.
The fact that the onset of shear thickening occurs at the same shear stress independent of the volume fraction of nonBrownian spheres suggests a reduction of the viscosity curves to a universal behavior using a single, vertical shift factor. Such a reduction is indeed possible, as shown in Fig. 8, which also plots the shift factor required to achieve a master curve as a function of the volume fraction of nonBrownian particles. These results for the steady shear viscosity in the present study are consistent with the hypothesis of Ohl and Gleissle [48]. This shift factor is the viscosity of the suspension relative to that of the colloidal dispersion medium (a type of relative viscosity) at constant shear stress. As such, it is tempting to compare this relative viscosity to that known for suspensions in Newtonian fluids [given by Eq. (1) with $\mathrm{k}_{2}=5.0$ ]. To a first approximation, the equations derived for particles in a Newtonian fluid can be used to estimate the viscosity in this concentration regime, although the relative steady shear viscosities measured in this study clearly lie above these predictions. Similar behavior has been observed with suspensions in other viscoelastic media such as Boger fluids [67], silicone oil [68], and polydimethylsiloxane [64] over a similar range of particle concentrations.

\section{Extension to higher particle concentrations}

For particle suspensions at higher particle concentrations, it is known that many-body interactions become important in determining the suspension viscosity, such that Eq. (1) is no longer applicable. The semiempirical model of Morris and Boulay [6] has been demonstrated to be successful at describing the viscosity of suspensions of non-Brownian spheres in a Newtonian fluid

$$
\eta_{r}=1+2.5 \phi\left(1-\frac{\phi}{\phi_{\max }}\right)^{-1}+K_{s}\left(\frac{\phi}{\phi_{\max }}\right)^{2}\left(1-\frac{\phi}{\phi_{\max }}\right)^{-2} .
$$
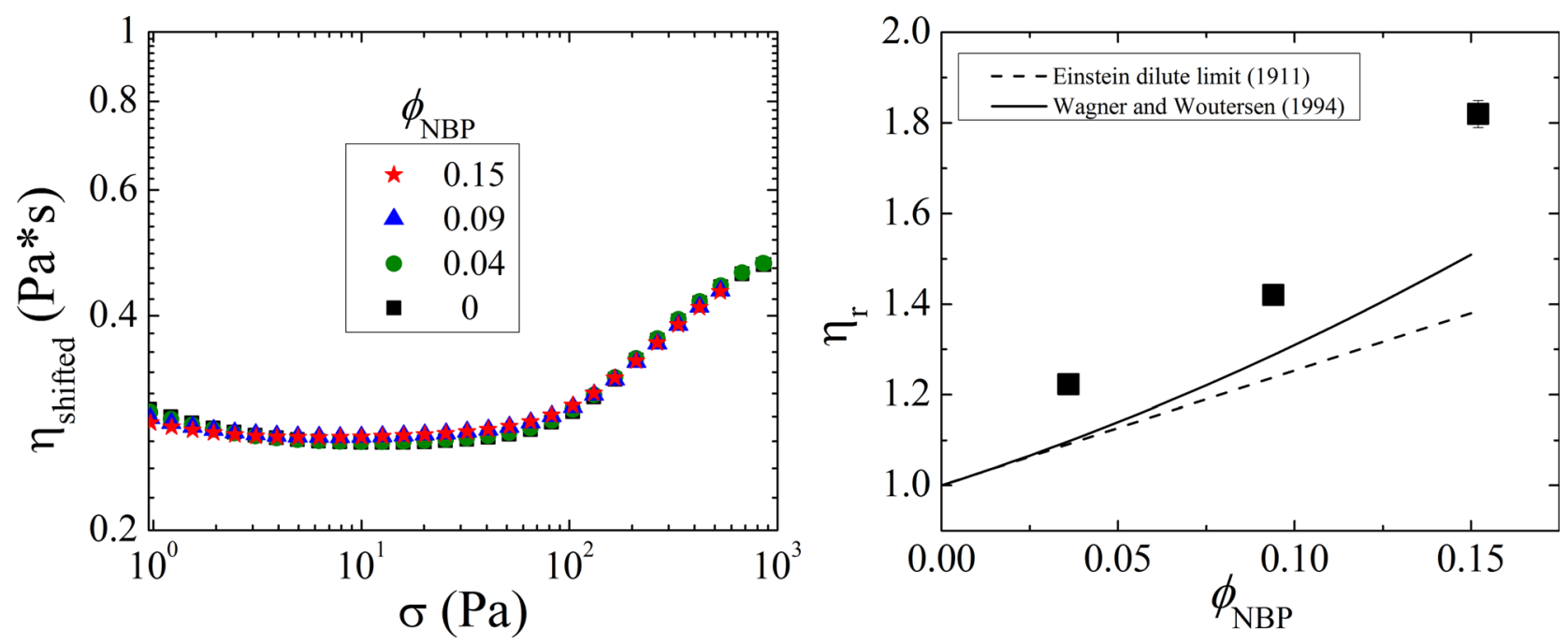

FIG. 8. (Left) Shifted viscosity curves from Fig. 7 for suspensions of non-Brownian spheres in a colloidal shear thickening fluid. (Right) Relative steady shear viscosity for suspensions in a colloidal dispersion medium as a function of the concentration of non-Brownian spheres. The dashed line is the Einstein viscosity equation [Eq. (1), $\mathrm{k}_{2}=0$ ] for dilute suspensions of non-Brownian spheres in a Newtonian fluid and the solid line contains the additional $\phi^{2}$ term of $\mathrm{k}_{2}=5.0$ [4]. Error bars are smaller than data points. 


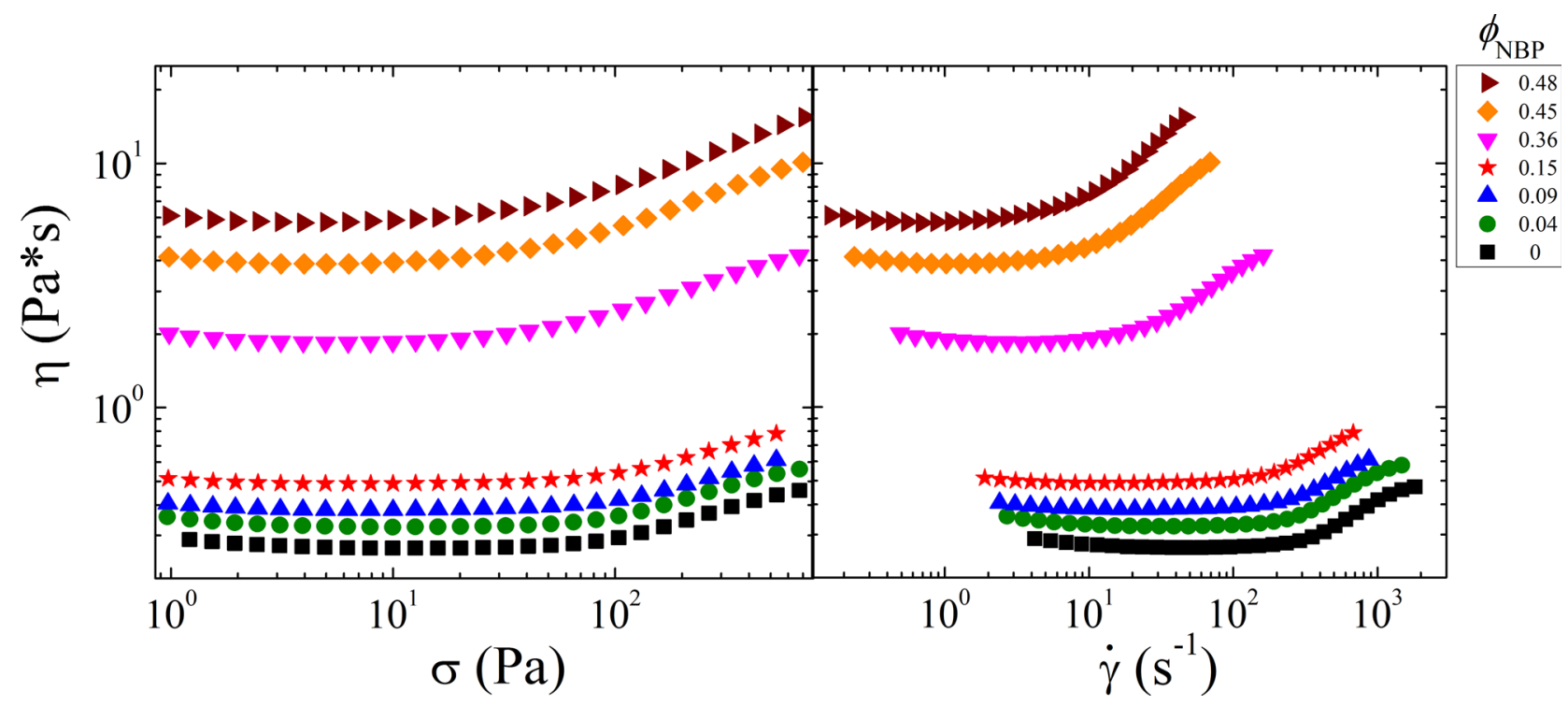

FIG. 9. Flow curves of suspensions of non-Brownian particles in a concentrated colloidal dispersion as a function of the shear stress (left) and shear rate (right).

In the equation above, $\phi_{\max }$ is the maximum packing fraction and $K_{s}$ is an adjustable fitting parameter.

The flow curves for suspensions containing high volume fractions of non-Brownian spheres are presented in Fig. 9. The high shear viscosities are taken at $10 \mathrm{~Pa}$ and are compared with the model of Morris and Boulay [6] in Fig. 10. This semiempirical model can describe the shift in relative viscosity in the high shear plateau with $\phi_{\max }$ equal to 0.58 , which has been commonly reported as the maximum packing fraction in a number of suspensions of non-Brownian spherical particles in Newtonian fluids [69-72]. This value of $\phi_{\max }$ is below the expected value of 0.638 for random close packing of monodisperse spheres. As our particles are polydisperse, the maximum packing fraction is expected to be larger, and the discrepancy may reflect weak attractive interactions, particle roughness, or particle shape anisotropy. Nonetheless, this model developed for suspensions in Newtonian fluids yields an excellent description of the suspension viscosity data in the high shear plateau at $\sigma=10 \mathrm{~Pa}$. A recent study [23] on suspensions of non-Brownian particles in yield stress emulsions also found the semiempirical model of Morris and Boulay [6] captured the viscosity data at high particle loadings. This particular model, originally proposed for particles in Newtonian fluids, has now found an unanticipated level of success at describing the behavior of suspensions in different types of viscoelastic media.

At these higher concentrations of non-Brownian particles, a single, vertical shift factor cannot produce a master curve for all shear stresses and deviations from the shifting procedure become apparent in the shear thickening regime, as illustrated in Fig. 10. Interestingly, the viscosity in the shear thickening regime increases more rapidly for higher concentrations of non-Brownian particles, as shown in Figs. 9 and 10. Recall that the particle Reynolds number for these suspensions is on the order of $10^{-3}$ in the shear thickening regime, such that particle inertia is not contributing to the measured viscosity.
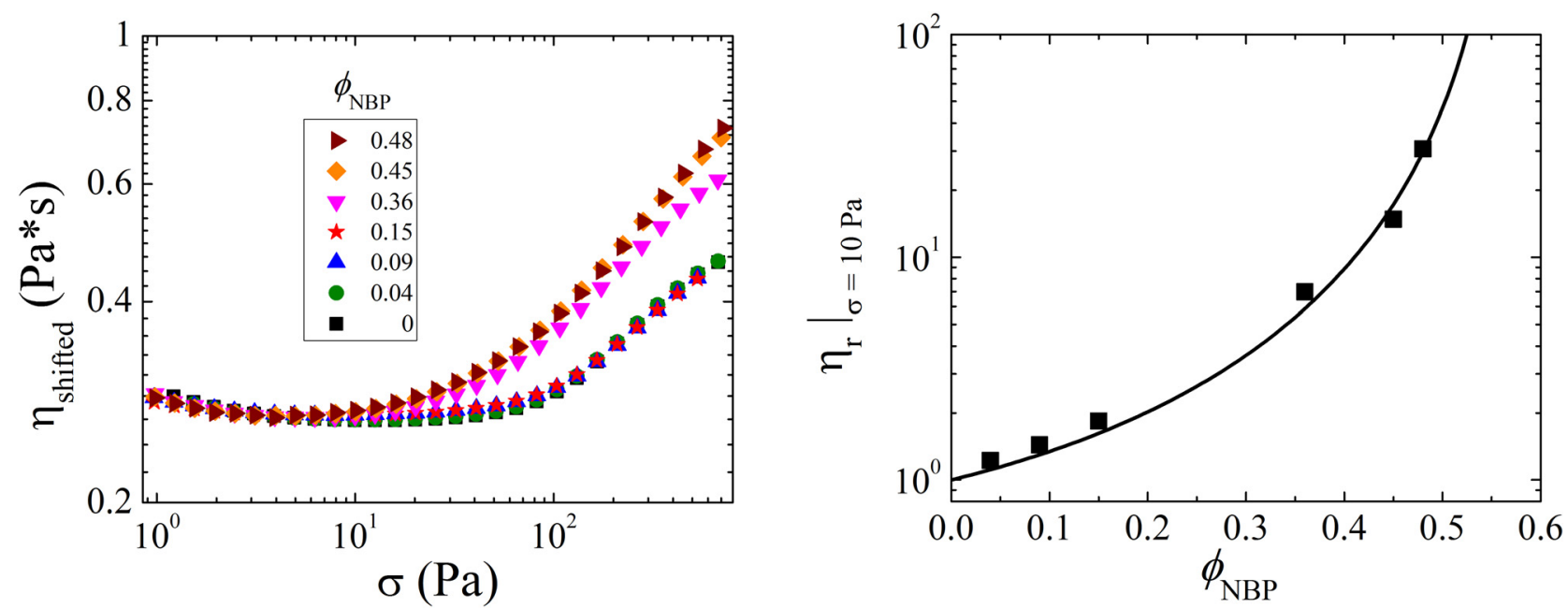

FIG. 10. (Left) Viscosity curves for suspensions shifted onto the high shear plateau of the colloidal dispersion medium at $\sigma=10$ Pa. (Right) Suspension viscosity relative to that of the colloidal dispersion at $\sigma=10 \mathrm{~Pa}$. The solid line is a fit of the semiempirical model of Morris and Boulay [6] with $K_{s}=0.94$. 


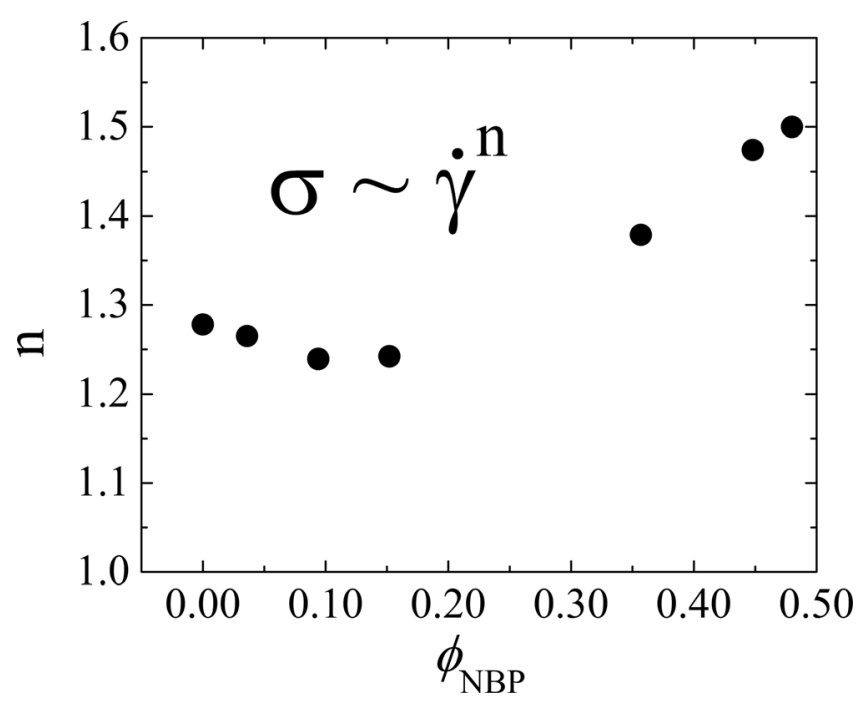

FIG. 11. Shear thickening power law exponent as a function of the volume fraction of non-Brownian spheres. Error bars are smaller than data points.

To quantitatively determine the power law exponent, we fit the data for each volume fraction in the range of $\sigma=100$ to $400 \mathrm{~Pa}$ to the power law given by

$$
\sigma=k \dot{\gamma}^{n}
$$

where $k$ is a constant and $n$ is the power law exponent. In this range of shear stresses, all of the dispersions exhibit a power-law shear thickening upturn before the viscosity begins tend toward the constant viscosity plateau of the shear-thickened state. The shear thickening power law exponent in this regime is shown to be an increasing function of the volume fraction of non-Brownian spheres in Fig. 11.

To understand this increase in the shear thickening power law exponent, it is useful to consider the average separation distance between non-Brownian spherical surfaces in suspension as a function of the volume fraction. A simple geometric model for this average separation distance is $[36,73]$

$$
\frac{h}{2 a}=\left(\frac{\phi_{\max }}{\phi}\right)^{1 / 3}-1
$$

where $h$ is the characteristic separation distance of particle surfaces, $a$ is the particle radius, $\phi$ is the particle volume fraction, and $\phi_{\max }$ is the maximum packing fraction, which is taken to be 0.58 to be consistent with the previous modeling. Figure 12 shows the shear thickening power law exponent as a function of the separation distance between nonBrownian particles $\left(h_{\mathrm{NBP}}\right)$ normalized by the diameter of the colloidal particles ( $\left.2 a_{\text {colloids }}\right)$. This parameter physically represents the number of colloidal particles that could span the characteristic gap between non-Brownian spherical surfaces.

Geometric confinement of a colloidal fluid is known to enhance shear thickening as demonstrated by the narrow-gap Couette measurements of Chow and Zukoski [74] and recent simulation studies of suspensions under confinement [75]. In both studies, gap confinement of order 10 particle diameters increased the shear thickening power law exponent. The change in the shear thickening power law exponent for our

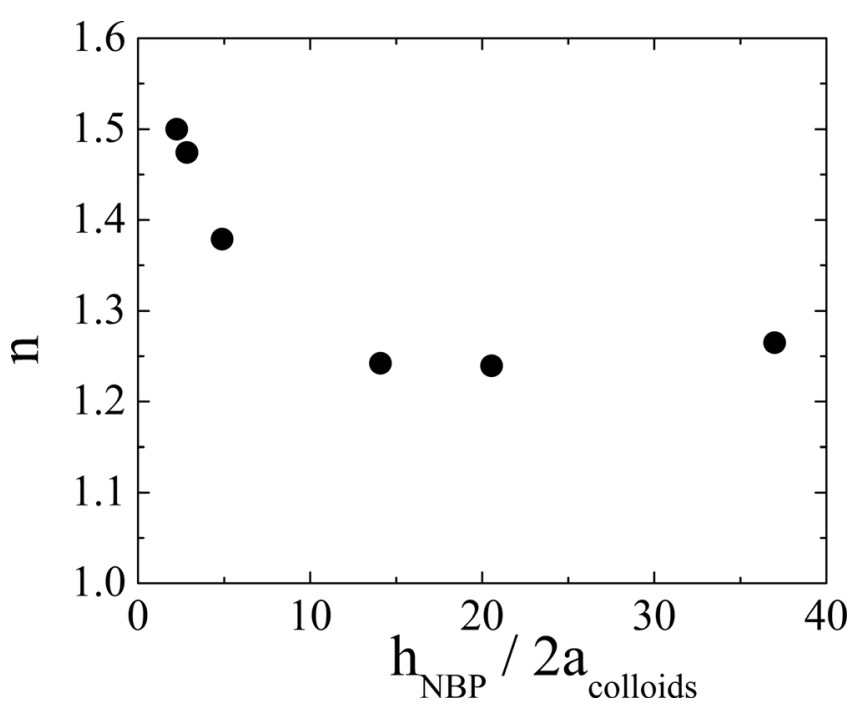

FIG. 12. Shear thickening power law exponent " $n$ " from Eq. (10) as a function of the characteristic separation distance between non-Brownian spheres as calculated from Eq. (11).

suspensions suggests that confinement of the colloidal dispersion medium between non-Brownian spherical surfaces may become relevant when the characteristic gap reduces to approximately ten times the diameter of the colloidal particles, as observed in Fig. 12. Below this average particle surface-to-surface spacing, the power law exponent increases. In our work, the large non-Brownian spheres provide a type of local confinement of the suspending colloidal fluid. Under shear flow, the colloidal fluid is forced to flow within the narrow gaps between the non-Brownian spheres. Thus, it is reasonable to anticipate that this local confinement can promote the formation of larger hydroclusters and stronger shear thickening. Note that this confinement between spherical non-Brownian particles is much less constrained geometrically than for parallel plates as in the simulations. Consequently, the confinement effects in suspension are not expected to be as dramatic as for confinement between walls. Furthermore, this enhancement in shear thickening is distinguishable from any changes in the molecular suspending fluid due to confinement, which can lead to increases in the viscosity when liquids are confined to gaps approaching the molecular size [76]. For example, Lee and Wagner [77] demonstrated that continuum hydrodynamics are maintained for colloidal dispersions in molecular liquids down to surfaceto-surface length scales of only a few nanometers.

\section{Normal stress differences}

Negative normal stress differences have been reported by authors for a number of suspensions consisting of nonBrownian spheres in Newtonian fluids [69,78-80]. Negative normal stress differences have also been observed in shear thickening colloidal dispersions, both in Stokesian dynamics simulations [81] and experimental measurements [82,83]. Cwalina and Wagner [7] recently reported measurements of the first and second normal stress differences for a model shear thickening colloidal dispersion of near hard spheres. Both $N_{1}$ and $N_{2}$ were found to be negative in the shear- 
thickened state with $\left|N_{2}\right|$ slightly larger than that of $\left|N_{1}\right|$ for the most concentrated dispersions. The normal stress differences in colloidal dispersions are a consequence of the highly anisotropic microstructure that develops under flow at high $P e$ due to lubrication hydrodynamic interactions between particles [55].

An extensive literature also exists for the measurement of normal stress differences in suspensions of non-Brownian spheres in viscoelastic media $[39,40,42,46,47,68,67]$. The normal stress differences exhibit power law behavior when plotted against the shear stress on logarithmic axes and shift parallel to each other as the particle concentration is varied. It is important to note that the normal stress differences in these suspensions arise from the elasticity of the underlying suspending fluid whereas the normal stress differences measured for suspensions in Newtonian fluids are a consequence of viscous hydrodynamic forces acting between particles.

Measurement of the normal stress differences, $\mathrm{N}_{1}-\mathrm{N}_{2}$, for the suspensions of non-Brownian particles in the colloidal dispersion of KE-P50 particles in PEG-200 are shown in Fig. 13. For guidance on the expected magnitude of $\mathrm{N}_{1}-\mathrm{N}_{2}$ for the colloidal dispersion, we used the semiempirical model of Morris and Boulay [6] along with measurements and model coefficients determined by Cwalina and Wagner [7]

$$
\mathrm{N}_{1}-\mathrm{N}_{2}=\left(\mathrm{K}_{2}-\mathrm{K}_{1}\right)\left(\frac{\phi}{\phi_{\max }}\right)^{2}\left(1-\frac{\phi}{\phi_{\max }}\right)^{-2} \eta_{f} \dot{\gamma}
$$

The values of $\mathrm{K}_{1}$ and $\mathrm{K}_{2}$ were reported by Cwalina and Wagner [7] to be 0.177 and 0.240 , respectively, in the shearthickened state. This limiting behavior of $\mathrm{N}_{1}-\mathrm{N}_{2}$ in the colloidal shear-thickened state for $\phi=0.40$ is shown in Fig. 13 as the solid line. This can be compared to the data for the colloidal dispersion, which is largely below the value of instrument resolution except at the highest shear rates. This

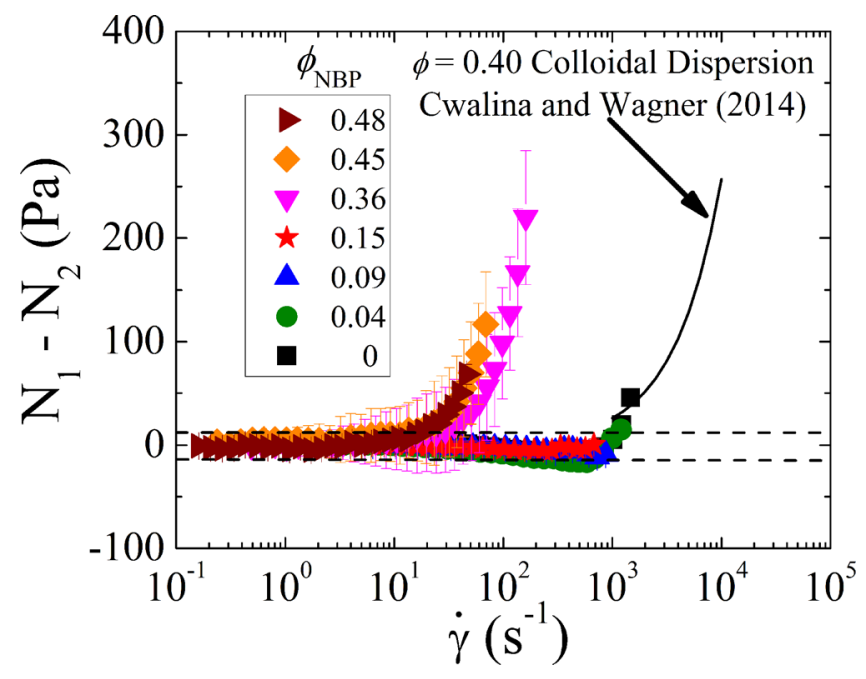

FIG. 13. Measurements of $N_{1}-N_{2}$ over a broad range of suspension volume fractions plotted against the shear rate. The solid line is the predicted value of $\mathrm{N}_{1}-\mathrm{N}_{2}$ for the colloidal dispersion medium $\left(\phi_{\mathrm{NBP}}=0\right)$ in the shearthickened state using the semiempirical model of Morris and Boulay [6] with the model parameters reported by Cwalina and Wagner [7]. The dashed lines mark the resolution of the instrument. high shear rate data compares well with model predictions for the limiting value. The parallel plate tooling limits the attainment of higher shear rates due to sample ejection.

In the semidilute regime, the addition of non-Brownian particles does not appreciably alter the values of $\mathrm{N}_{1}-\mathrm{N}_{2}$ to within instrument resolution, which is denoted by the dashed lines in Fig. 13. This is consistent with measurements on suspensions in other viscoelastic media, where the normal stress differences are largely due to the underlying suspending fluid at comparable shear rates [40,60]. However, at higher volume fractions, large positive values of $\mathrm{N}_{1}-\mathrm{N}_{2}$ are measured that greatly exceed those of the colloidal fluid itself. The measured normal stress differences for the suspensions are replotted in Fig. 14 as a function of the shear stress. A semi-empirical prediction can be made for the scaling of the normal stress differences in the shear-thickened state by using Eq. (12) and dividing both sides by the shear stress to obtain

$$
\frac{\mathrm{N}_{1}-\mathrm{N}_{2}}{\sigma}=\left(\mathrm{K}_{2}-\mathrm{K}_{1}\right)\left(\frac{\phi}{\phi_{\max }}\right)^{2}\left(1-\frac{\phi}{\phi_{\max }}\right)^{-2} \eta_{f} \frac{\dot{\gamma}}{\sigma} .
$$

The right-hand side of Eq. (13) can be rewritten as

$$
\frac{\mathrm{N}_{1}-\mathrm{N}_{2}}{\sigma}=\left(\mathrm{K}_{2}-\mathrm{K}_{1}\right)\left(\frac{\phi}{\phi_{\max }}\right)^{2}\left(1-\frac{\phi}{\phi_{\max }}\right)^{-2} \frac{1}{\eta_{r}} .
$$

The measurements of Cwalina and Wagner [7] can be used with Eq. (9) to obtain the predicted relative viscosity in the shear-thickened state as: $\left(\mathrm{N}_{1}-\mathrm{N}_{2}\right) / \sigma=0.046$. This scaling is shown by the dashed line in Fig. 14 and it captures the observed behavior of the underlying colloidal fluid to a first approximation. In our recent paper [7], we argue that the

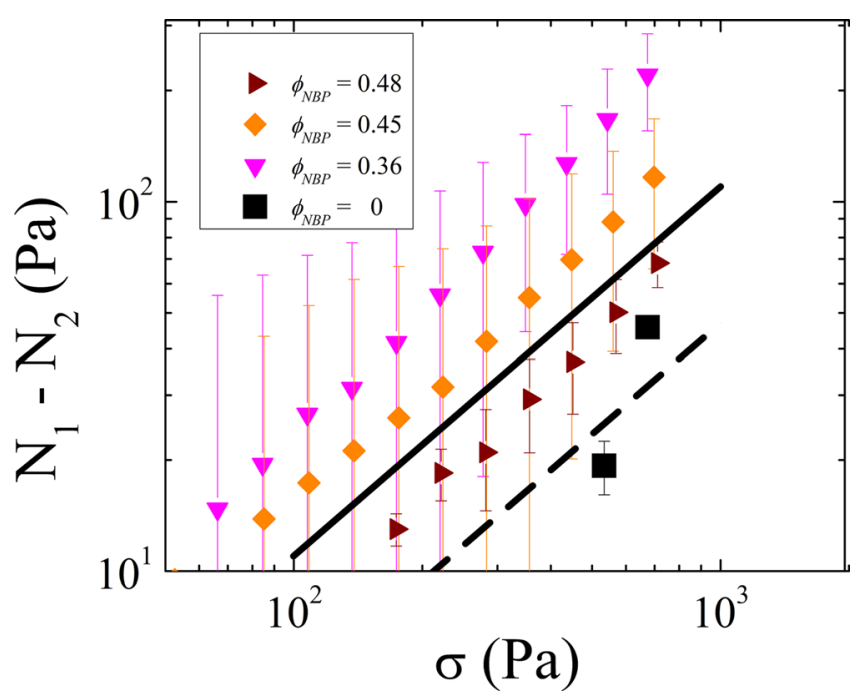

FIG. 14. Measured normal stress differences plotted as a function of the shear stress. Only data points lying above the instrument resolution are shown. The dashed line is the expected ratio of the normal stress differences to the shear stress for the underlying colloidal dispersion $\left(\phi_{\mathrm{NBP}}=0\right)$ in the shear-thickened state [7]. The solid line is the ratio of the normal stress differences to the shear stress for the underlying colloidal fluid in the limit $\left(\phi / \phi_{\max }\right) \rightarrow 1$, which corresponds to the enhancement of shear thickening in the suspending medium due to confinement between non-Brownian particle surfaces at high packing fractions. 
value of the relative viscosity in the shear-thickened state increases with the particle volume fraction because the system is closer to maximum packing due to the formation of hydroclusters with an effective volume fraction larger than just that of the constituent solid particles $[84,85]$. In Sec. IIIC, the increase in the shear thickening power law exponent at high packing fractions of non-Brownian particles was attributed to confinement of the colloidal fluid between particle surfaces, which promotes the formation of locally larger hydroclusters. Thus, if we adopt the consistent viewpoint that the formation of larger hydroclusters raised the effective particle concentration in the colloidal fluid $\left(\left(\phi / \phi_{\max }\right) \rightarrow 1\right)$, the modeling of Cwalina and Wagner [7] predicts the ratio of the normal stress differences to the shear stress in the shear-thickened state for this $\phi=0.40$ colloidal fluid should be given by $\left(\mathrm{N}_{1}-\mathrm{N}_{2}\right) / \sigma=0.11$. This scaling is shown by the solid line in Fig. 14. There is a large experimental uncertainty in the data at high packing fraction of non-Brownian particles. However, to a first approximation, this scaling captures the behavior of the normal stress differences relative to the shear stress. This scaling of the normal stress differences suggests the origin is from the colloidal fluid and it further supports the hypothesis of confinement at high packing fractions of non-Brownian particles.

\section{E. Comparison to other scaling theories}

We conclude the discussion with a comparison of the present work to the scaling theories proposed by Liard et al. [22] for suspensions in generalized Newtonian fluids. In their work, they explore a sequence of shifts that is formally equivalent to the stress scaling of Highgate and Whorlow [39], Barnes [86], and Gleissle and Hochstein [87]. However, the authors were not able to achieve as successful a reduction of the data to a master curve as shown here and in aforementioned references. Indeed, the authors indicated that the viscosity shift factors were higher for the shear thickening regime than the shear thinning portion of their data. The deviations from a simple stress scaling were more apparent at higher particle volume fractions. The authors identified a different scaling with volume fraction between the distribution of local shear rates and the bulk hydrodynamic viscosity as the likely source of this violation of the simple scaling law for suspensions in generalized Newtonian fluids. Here, we propose a new mechanism for violation of this simple stress scaling law for suspensions dispersed in colloidal shear thickening fluids. Namely, confinement between the surfaces of non-Brownian particles at high packing fractions leads to enhanced shear thickening of the colloidal dispersion. This effect is specific to the use of a colloidal dispersion as the suspending medium and the possible role of suspension particle shape in enhancing or mitigating this confinement is to be explored further in the future.

\section{CONCLUSIONS}

Measurements of the dynamic and steady shear rheology for model suspensions comprised of non-Brownian spheres suspended in a non-Newtonian medium, itself comprised of a colloidal shear thickening dispersion, are found to strongly reflect the underlying rheological properties of the colloidal dispersion. The linear viscoelasticity of these suspensions is found to mimic that of the suspending colloidal dispersion such that the characteristic relaxation time of the suspension remains unchanged upon the addition of non-Brownian spherical particles. In a similar manner, for low volume fractions of non-Brownian spheres, the steady shear viscosity curves are found to superimpose through a vertical shift when plotted against the shear stress. This behavior has been reported for suspensions in molecular non-Newtonian fluids and is consistent with the hypothesis of Ohl and Gleissle [48]. Deviations from this simple shifting are observed at higher volume fractions of non-Brownian particles at high shear stresses, where the shear thickening power law exponent is found to increase with the addition of non-Brownian spheres. This violation of the simple scaling can be attributed to the known effect of confinement on enhancing shear thickening in colloidal dispersions [74], where the confinement is due to the packing of the larger non-Brownian particles. The normal stress differences are significantly enhanced at high packing fractions and their scaling with the shear stress is consistent with confinement of the underlying colloidal dispersion by the non-Brownian spheres, which enhances shear thickening in the colloidal suspending medium.

This experimental investigation of model suspensions of non-Brownian spherical particles suspended in a nonNewtonian colloidal dispersion reveals a relatively simple and predictable rheological response for shear stresses corresponding to the shear thinning regime and high shear plateau of the colloidal dispersion. However, when shear thickening is evident in the colloidal dispersions, confinement effects become evident at a higher concentration of non-Brownian spheres. Such confinement effects may be even further enhanced in the presence of discontinuous shear thickening colloidal dispersions, and future work will address this as well as the role of particle shape.

We note that recent work on novel shear thickening suspoemulsions by Fowler et al. [34] has also shown unanticipated effects in emulsion morphology when the colloidal dispersion is in the shear-thickened state. Further, the present work complements previous investigations of non-Brownian particles in non-Newtonian media such as shear thinning polymer solutions and melts, wormlike micelles, or Boger fluids where particles have been observed to chain along the flow direction [31-33]. The results presented here motivate the need for further exploration of the behavior of suspended non-Brownian particles in a shear thickening colloidal dispersion.

\section{ACKNOWLEDGMENTS}

This work was supported by a NASA EPSCoR Grant (NNX11AQ28A) and a Delaware Space Grant Graduate Fellowship (NNX10AN63H).

\section{APPENDIX: MODIFIED CROSS MODEL}

As shown in Fig. 1, a modified Cross model given by Galindo-Rosales et al. [58] is used to extract the zero-shear and high shear plateau viscosities, which are then compared 
TABLE III. Modified Cross model parameters used in Fig. 1.

\begin{tabular}{lc}
\hline \hline Parameter (Units) & Value \\
\hline$\eta_{0}\left(\mathrm{~Pa}^{*} \mathrm{~s}\right)$ & 11.66 \\
$\eta_{\mathrm{c}}\left(\mathrm{Pa}^{*} \mathrm{~s}\right)$ & 0.28 \\
$\eta_{\max }\left(\mathrm{Pa}^{*} \mathrm{~s}\right)$ & 0.47 \\
$\mathrm{n}_{1}$ & 0.36 \\
$\mathrm{n}_{2}$ & 0.83 \\
$\lambda_{1}\left(\mathrm{~s}^{)}\right.$ & $2.13 * 10^{7}$ \\
$\lambda_{2}(\mathrm{~s})$ & $3.76 * 10^{-3}$ \\
$\dot{\gamma}_{c}\left(\mathrm{~s}^{-1}\right)$ & 234.45 \\
$\dot{\gamma}_{\max }\left(\mathrm{s}^{-1}\right)$ & 1821.17 \\
\hline \hline
\end{tabular}

to the expected limiting hard-sphere behavior. The model of Galindo-Rosales et al. [58] divides the viscosity curve into two regions. Region 1 models the viscosity as a function of the shear rate up to the critical shear rate for shear thickening as follows:

$$
\eta_{1}(\dot{\gamma})=\eta_{c}+\frac{\eta_{0}-\eta_{c}}{1+\left[\lambda_{1}\left(\frac{\dot{\gamma}^{2}}{\dot{\gamma}_{c}-\dot{\gamma}}\right)\right]^{n_{1}}},
$$

where $\eta_{0}$ and $\eta_{c}$ are the respective values of the zero-shear viscosity and the viscosity at the critical shear rate, $\dot{\gamma}_{c}$. Only $\eta_{0}$ is extracted from the fit as $\eta_{c}$ is a fixed value taken from the data. $\lambda_{1}$ and $n_{1}$ are adjustable fitting parameters. In Region 2, the viscosity data are fit from the critical shear rate up to the maximum shear rate attained in the shear-thickened state

$$
\eta_{2}(\dot{\gamma})=\eta_{\max }+\frac{\eta_{c}-\eta_{\max }}{1+\left[\lambda_{2}\left(\frac{\dot{\gamma}-\dot{\gamma}_{c}}{\dot{\gamma}_{\max }-\dot{\gamma}}\right) \dot{\gamma}\right]^{n_{2}}},
$$

where $\eta_{\max }$ is the value of the viscosity at the maximum shear rate in the shear-thickened state, $\dot{\gamma}_{\max }$. Both of these values are fixed values extracted from the data set. $\lambda_{2}$ and $n_{2}$ are adjustable fitting parameters. All model parameters, both fixed and adjustable, are reported in Table III for Regions 1 and 2.

\section{References}

[1] Mewis, J., and N. J. Wagner, Colloidal Suspension Rheology (Cambridge University, New York, 2012).

[2] Einstein, A., "Berichtigung zu meiner Arbeit: "Eine neue Bestimmung der Molek ldimensionen”," Annalen der Physik. 339, 591-592 (1911).

[3] Batchelor, G. K., and J. T. Green, "Determination of bulk stress in a suspension of spherical-particles to order C-2," J. Fluid Mech. 56, 401-427 (1972).

[4] Wagner, N. J., and A. T. J. M. Woutersen, "The viscosity of bimodal and polydisperse suspensions of hard-spheres in the dilute limit," J. Fluid Mech. 278, 267-287 (1994).

[5] Faroughi, S. A., and C. Huber, "A generalized equation for rheology of emulsions and suspensions of deformable particles subjected to simple shear at low Reynolds number," Rheol. Acta 54, 85-108 (2015).

[6] Morris, J. F., and F. Boulay, "Curvilinear flows of noncolloidal suspensions: The role of normal stresses," J. Rheol. 43, 1213-1237 (1999).
[7] Cwalina, C. D., and N. J. Wagner, "Material properties of the shearthickened state in concentrated near hard-sphere colloidal dispersions," J Rheol. 58, 949-967 (2014).

[8] Brady, J. F., and J. F. Morris, "Microstructure of strongly sheared suspensions and its impact on rheology and diffusion," J. Fluid Mech. 348, 103-139 (1997).

[9] Russel, W. B., N. J. Wagner, and J. Mewis, "Divergence in the low shear viscosity for Brownian hard-sphere dispersions: At random close packing or the glass transition?," J. Rheol. 57, 1555-1567 (2013).

[10] Farr, R. S., and R. D. Groot, "Close packing density of polydisperse hard spheres," J. Chem. Phys. 131, 244104 (2009).

[11] Shewan, H. M., and J. R. Stokes, "Analytically predicting the viscosity of hard sphere suspensions from the particle size distribution," J. NonNewtonian Fluid Mech. 222, 72-81 (2015).

[12] Mari, R., R. Seto, J. F. Morris, and M. M. Denn, "Shear thickening, frictionless and frictional rheologies in non-Brownian suspensions," J. Rheol. 58, 1693-1724 (2014).

[13] Haddadi, H., and J. F. Morris, "Microstructure and rheology of finite inertia neutrally buoyant suspensions," J. Fluid Mech. 749, 431-459 (2014).

[14] Ovarlez, G., S. Rodts, X. Chateau, and P. Coussot, "Phenomenology and physical origin of shear localization and shear banding in complex fluids," Rheol. Acta 48, 831-844 (2009).

[15] Aitcin, P. C., "Cements of yesterday and today-Concrete of tomorrow," Cem. Concr. Res. 30, 1349-1359 (2000).

[16] Toussaint, F., C. Roy, and P. H. Jezequel, "Reducing shear thickening of cement-based suspensions," Rheol. Acta 48, 883-895 (2009).

[17] Zapata, P., and J. A. Gambatese, "Energy consumption of asphalt and reinforced concrete pavement materials and construction," J. Infrastruct. Syst. 11, 9-20 (2005).

[18] Mishra, S. K., P. K. Senapati, and D. Panda, "Rheological behavior of coal-water slurry," Energy Sources 24, 159-167 (2002).

[19] Chen, L. Y., Y. F. Duan, C. S. Zhao, and L. G. Yang, "Rheological behavior and wall slip of concentrated coal water slurry in pipe flows," Chem. Eng. Process. 48, 1241-1248 (2009).

[20] Nguyen, Q. D., and D. V. Boger, "Application of rheology to solving tailings disposal problems," Int. J. Miner. Process. 54, 217-233 (1998).

[21] Goff, H. D., "Colloidal aspects of ice cream-A review," Int. Dairy J. 7, 363-373 (1997).

[22] Liard, M., N. S. Martys, W. L. George, D. Lootens, and P. Hebraud, "Scaling laws for the flow of generalized Newtonian suspensions," J. Rheol. 58, 1993-2015 (2014).

[23] Dagois-Bohy, S., S. Hormozi, E. Guazzelli, and O. Pouliquen, "Rheology of dense suspensions of non-colloidal spheres in yieldstress fluids," J. Fluid Mech. 776, R2 (2015).

[24] Slattery, J. C., and R. B. Bird, "Non-Newtonian flow past a sphere," Chem. Eng. Sci. 16, 231-241 (1961).

[25] Karnis, A., and S. G. Mason, "Particle motions in non-Newtonian media,” Trans. Soc. Rheol. 10, 571-592 (1966).

[26] Gauthier, F., H. L. Goldsmith, and S. G. Mason, "Particle motions in nonNewtonian media: I. Couette flow,” Rheol Acta. 10, 344-364 (1971).

[27] D’Avino, G., M. A. Hulsen, F. Snijkers, J. Vermant, F. Greco, and P. L. Maffettone, "Rotation of a sphere in a viscoelastic liquid subjected to shear flow. Part I: Simulation results," J. Rheol. 52, 1331-1346 (2008).

[28] D'Avino, G., P. L. Maffettone, M. A. Hulsen, and G. W. M. Peters, "Numerical simulation of planar elongational flow of concentrated rigid particle suspensions in a viscoelastic fluid," J. Non-Newtonian Fluid Mech. 150, 65-79 (2008).

[29] Snijkers, F., G. D’Avino, P. L. Maffettone, F. Greco, M. Hulsen, and J. Vermant, "Rotation of a sphere in a viscoelastic liquid subjected to 
shear flow. Part II. Experimental results,” J. Rheol. 53, 459-480 (2009).

[30] Fabris, D., S. J. Muller, and D. Liepmann, "Wake measurements for flow around a sphere in a viscoelastic fluid," Phys. Fluids 11, 3599-3612 (1999).

[31] Michele, J., R. Patzold, and R. Donis, "Alignment and aggregation effects in suspensions of spheres in non-Newtonian media," Rheol. Acta. 16, 317-321 (1977).

[32] Lyon, M. K., D. W. Mead, R. E. Elliott, and L. G. Leal, "Structure formation in moderately concentrated viscoelastic suspensions in simple shear flow," J. Rheol. 45, 881-890 (2001).

[33] Scirocco, R., J. Vermant, and J. Mewis, "Effect of the viscoelasticity of the suspending fluid on structure formation in suspensions," J. NonNewtonian Fluid Mech. 117, 183-192 (2004).

[34] Fowler, J. N., J. Kirkwood, and N. J. Wagner, "Rheology and microstructure of shear thickening fluid suspoemulsions," Appl. Rheol. 24, 16-25 (2014).

[35] Flatt, R. J., N. Martys, and L. Bergstrom, "The rheology of cementitious materials," MRS Bull. 29, 314-318 (2004).

[36] Bender, J., and N. J. Wagner, "Reversible shear thickening in monodisperse and bidisperse colloidal dispersions," J. Rheol. 40, 899-916 (1996).

[37] Chong, J. S., E. B. Christiansen, and A. D. Baer, "Rheology of concentrated suspensions," J. Appl. Polym. Sci. 15, 2007-2021 (1971).

[38] Shapiro, A. P., and R. F. Probstein, "Random packings of spheres and fluidity limits of monodisperse and bidisperse suspensions," Phys. Rev. Lett. 68, 1422-1425 (1992).

[39] Highgate, D. J., and R. W. Whorlow, "Rheological properties of suspensions of spheres in non-Newtonian media," Rheol Acta 9, 569-576 (1970).

[40] Mewis, J., and R. de Bleyser, "Concentration effects in viscoelastic dispersions," Rheol Acta. 14, 721-728 (1975).

[41] Kataoka, T., T. Kitano, M. Sasahara, and K. Nishijima, "Viscosity of particle filled polymer melts," Rheol Acta. 17, 149-155 (1978).

[42] Chan, D., and R. L. Powell, "Rheology of suspensions of spherical particles in a Newtonian and a non-Newtonian fluid," J. Non-Newtonian Fluid Mech. 15, 165-179 (1984).

[43] Metzner, A. B., "Rheology of suspensions in polymeric liquids," J. Rheol. 29, 739-775 (1985).

[44] Poslinski, A. J., M. E. Ryan, R. K. Gupta, S. G. Seshadri, and F. J. Frechette, "Rheological behavior of filled polymeric systems. 1. Yield stress and shear-thinning effects," J. Rheol. 32, 703-735 (1988).

[45] Malkin, A. Y., "Rheology of filled polymers," Adv. Polym. Sci. 96, 69-97 (1990).

[46] Aral, B. K., and D. M. Kalyon, "Viscoelastic material functions of noncolloidal suspensions with spherical particles," J Rheol. 41, 599-620 (1997).

[47] Zarraga, I. E., D. A. Hill, and D. T. Leighton, "Normal stresses and free surface deformation in concentrated suspensions of noncolloidal spheres in a viscoelastic fluid," J. Rheol. 45, 1065-1084 (2001).

[48] Ohl, N., and W. Gleissle, "The characterization of the steady-state shear and normal stress functions of highly concentrated suspensions formulated with viscoelastic liquids," J. Rheol. 37, 381-406 (1993).

[49] Shikata, T., and D. S. Pearson, "Viscoelastic behavior of concentrated spherical suspensions," J. Rheol. 38, 601-616 (1994).

[50] Laun, H. M., "Rheological properties of aqueous polymer dispersions," Angew. Makromol. Chem. 123, 335-359 (1984).

[51] Morrison, F. A., Understanding Rheology (Oxford University, New York, 2001).

[52] Kalman, D. P., "Microstructure and rheology of concentrated suspensions of near hard-sphere colloids," Ph.D. thesis, University of Delaware, Delaware, 2010.
[53] Gurnon, A. K., and N. J. Wagner, "Microstructure and rheology relationships for shear thickening colloidal dispersions," J. Fluid Mech. 769, 242-276 (2015).

[54] Russel, W. B., D. A. Saville, and W. R. Schowalter, Colloidal Dispersions (Cambridge University, New York, 1989).

[55] Bergenholtz, J., J. Brady, and M. Vicic, "The non-Newtonian rheology of dilute colloidal suspensions," J. Fluid Mech. 456, 239-275 (2002).

[56] Brady, J. F., "The rheological behavior of concentrated colloidal dispersions,” J. Chem. Phys. 99, 567-581 (1993).

[57] Dekruif, C. G., E. M. F. Vanlersel, A. Vrij, and W. B. Russel, "Hardsphere colloidal dispersions-Viscosity as a function of shear rate and volume fraction," J. Chem. Phys. 83, 4717-4725 (1985).

[58] Galindo-Rosales, F. J., F. J. Rubio-Hernandez, A. Sevilla, and R. H. Ewoldt, "How Dr. Malcom M. Cross may have tackled the development of "An apparent viscosity function for shear thickening fluids,"” J. Non-Newtonian Fluid Mech. 166, 1421-1424 (2011).

[59] Kulkarni, P. M., and J. F. Morris, "Suspension properties at finite Reynolds number from simulated shear flow," Phys. Fluids 20, 040602 (2008).

[60] Chan, D., and R. L. Powell, "Rheology of suspensions of sphericalparticles in a Newtonian and a non-Newtonian fluid," J. NonNewtonian Fluid Mech. 15, 165-179 (1984).

[61] Schaink, H. M., J. J. M. Slot, R. J. J. Jongschaap, and J. Mellema, "The rheology of systems containing rigid spheres suspended in both viscous and viscoelastic media, studied by Stokesian dynamics simulations," J. Rheol. 44, 473-498 (2000).

[62] See, H., P. Jiang, and N. Phan-Thien, "Concentration dependence of the linear viscoelastic properties of particle suspensions," Rheol. Acta 39, 131-137 (2000).

[63] Le Meins, J. F., P. Moldenaers, and J. Mewis, "Suspensions in polymer melts. 1. Effect of particle size on the shear flow behavior," Ind. Eng. Chem. Res. 41, 6297-6304 (2002).

[64] Pasquino, R., N. Grizzuti, P. L. Maffettone, and F. Greco, "Rheology of dilute and semidilute noncolloidal hard sphere suspensions," J. Rheol. 52, 1369-1384 (2008).

[65] Maranzano, B. J., and N. J. Wagner, "The effects of particle size on reversible shear thickening of concentrated colloidal dispersions," J. Chem. Phys. 114, 10514-10527 (2001).

[66] Shenoy, S. S., and N. J. Wagner, "Influence of medium viscosity and adsorbed polymer on the reversible shear thickening transition in concentrated colloidal dispersions," Rheol. Acta. 44, 360-371 (2005).

[67] Tanner, R. I., S. C. Dai, F. Z. Qi, and K. Housiadas, "Viscometric functions of semi-dilute non-colloidal suspensions of spheres in a viscoelastic matrix,” J. Non-Newtonian Fluid Mech. 201, 130-134 (2013).

[68] Mall-Gleissle, S. E., W. Gleissle, G. H. McKinley, and H. Buggisch, "The normal stress behaviour of suspensions with viscoelastic matrix fluids," Rheol. Acta 41, 61-76 (2002).

[69] Zarraga, I. E., D. A. Hill, and D. T. Leighton, "The characterization of the total stress of concentrated suspensions of noncolloidal spheres in Newtonian fluids (vol 44, pg 185, 2000)," J. Rheol. 44, 671-671 (2000).

[70] Singh, A., and P. R. Nott, "Experimental measurements of the normal stresses in sheared Stokesian suspensions," J. Fluid Mech. 490, 293-320 (2003).

[71] Boyer, F., E. Guazzelli, and O. Pouliquen, "Unifying suspension and granular rheology," Phys. Rev. Lett. 107, 188301 (2011).

[72] Tanner, R. I., F. Z. Qi, and S. C. Dai, "Scaling the normal stresses in concentrated non-colloidal suspensions of spheres," Rheol. Acta 52, 291-295 (2013).

[73] Boersma, W. H., J. Laven, and H. N. Stein, "Shear thickening (dilatancy) in concentrated dispersions," AIChE J. 36, 321-332 (1990). 
[74] Chow, M. K., and C. F. Zukoski, "Gap size and shear history dependencies in shear thickening of a suspension ordered at rest," J. Rheol. 39, 15-32 (1995).

[75] Bian, X., S. Litvinov, S. M. Ellero, and N. J. Wagner, "Hydrodynamic shear thickening of particulate suspension under confinement," J. NonNewtonian Fluid Mech. 213, 39-49 (2014).

[76] Granick, S., "Motions and relaxations of confined liquids," Science 253, 1374-1379 (1991).

[77] Lee, Y. S., and N. J. Wagner, "Rheological properties and small-angle neutron scattering of a shear thickening, nanoparticle dispersion at high shear rates," Ind. Eng. Chem. Res. 45, 7015-7024 (2006).

[78] Gadalamaria, F., and A. Acrivos, "Shear-induced structure in a concentrated suspension of solid spheres," J. Rheol. 24, 799-814 (1980).

[79] Couturier, E., F. Boyer, O. Pouliquen, and E. Guazzelli, "Suspensions in a tilted trough: Second normal stress difference," J. Fluid Mech. 686, 26-39 (2011).

[80] Dai, S. C., E. Bertevas, F. Z. Qi, and R. I. Tanner, "Viscometric functions for noncolloidal sphere suspensions with Newtonian matrices," J Rheol. 57, 493-510 (2013).
[81] Foss, D. R., and J. F. Brady, "Brownian dynamics simulation of hardsphere colloidal dispersions," J. Rheol. 44, 629-651 (2000).

[82] Laun, H. M., "Normal stresses in extremely shear thickening polymer dispersions," J. Non-Newtonian Fluid Mech. 54, 87-108 (1994).

[83] Lee, M., M. Alcoutlabi, J. J. Magda, C. Dibble, M. J. Solomon, X. Shi, and G. B. McKenna, "The effect of the shear-thickening transition of model colloidal spheres on the sign of N-1 and on the radial pressure profile in torsional shear flows," J. Rheol. 50, 293-311 (2006).

[84] Vand, V., "Viscosity of solutions and suspensions.1. Theory," J. Phys. Colloid Chem. 52, 277-299 (1948).

[85] O'Brien, V. T., and M. E. Mackay, "Stress components and shear thickening of concentrated hard sphere suspensions," Langmuir 16, 7931-7938 (2000).

[86] Barnes, H., "A review of the rheology of filled viscoelastic systems," Rheol. Rev. 2003, 1-36.

[87] Gleissle, W., and B. Hochstein, "Validity of the Cox-Merz rule for concentrated suspensions,” J. Rheol. 47, 897-910 (2003). 